\title{
AUTOMAÇÃO DE SISTEMA DE IRRIGAÇÃO COM MONITORAMENTO VIA APLICATIVO WEB
}

\author{
Gustavo Rissari Correia ${ }^{1}$, Helder Roberto de Oliveira Rocha ${ }^{2}$, Silvia das Dores Rissino ${ }^{3}$
}

\section{RESUMO}

Este trabalho apresenta uma proposta de desenvolvimento de um protótipo de baixo custo de aquisição (plataforma Arduino) para monitoramento e controle automático da irrigação, com acionamento remoto via aplicativo WEB. O trabalho consistiu na construção de dispositivo físico (hardware), software (acesso via WEB e para sistema operacional Android) e testes em campo. Utilizou-se de código aberto (open source), de equipamentos de automação e de instrumentos de comunicação remota. O sistema também conta com um conjunto de algoritmos que permitirá o melhor uso dos recursos hídricos a partir de informações coletadas de sensores inseridos no ambiente que estará sujeito à irrigação.

Palavras-Chave: agricultura, Android, Arduino, evapotranspiração, Wireless, Web.

\section{ABSTRACT}

\section{AUTOMATION IRRIGATION SYSTEM WITH MONITORING FROM A WEB APPLICATION}

This work presents a proposal to develop a prototype of low cost (Arduino platform) for monitoring and automatic control of irrigation, with remote activation via web application. The work consisted of building physical device (hardware), software (access via the web and Android OS) and field testing. Using open source (open source), automation equipment and remote communication tools. The system also has a set of algorithms that allow the best use of water resources from information collected from sensors embedded in the environment that will be subject to irrigation.

Keywords: agriculture, Android, Arduino, evapotranspiration, Wireless, Web.

\section{Recebido para publicação em 19/05/2015. Aprovado em 05/02/2016.}

1 - Engenheiro de Computação, gustavorissari@gmail.com

2 - Engenheiro Eletricista, Prof. Doutor da UFES/São Mateus-ES, helder.rocha@ufes.br

3 - Cientista de Computação, Prof. Doutora da UFES/São Mateus-ES, silvia.rissino@ufes.br

\section{REVENG




\section{INTRODUÇÃO}

A irrigação é uma técnica milenar que nos últimos anos tem-se desenvolvido acentuadamente, apresentando equipamentos e sistemas para as mais distintas condições. A história da irrigação se confunde com a do desenvolvimento e prosperidade econômica dos povos (Bernardo et al., 2006).

A agricultura irrigada tem sido importante estratégia para otimização da produção mundial de alimentos, proporcionando desenvolvimento sustentável no campo, com geração de empregos e renda de forma estável (Mantovani et al., 2009).

Com a aplicação de pouca água (irrigação com déficit), a produção não poderia obter o benefício esperado. Por sua vez, a aplicação excessiva de água é muito mais prejudicial, pois satura o solo, o que impede a sua aeração, lixivia nutrientes, induz maiores evaporação e salinização e, posteriormente, pode elevar o lençol freático para um nível que pode ser drenado somente a um alto custo. Além disso, o excesso propicia microclima favorável ao desenvolvimento de doenças, que podem causar prejuízo à cultura. As principais perdas diretas aos agricultores são o desperdício de fertilizantes: levados pela água em excesso que ultrapassa a profundidade das raízes, contaminando as fontes subterrâneas; aumento do custo da produção: maior consumo de energia para bombeamento da água; doenças provocadas por fungos e falta de aeração nas raízes: o excesso de água promove a proliferação de fungos no solo e nas raízes, bem como a redução da aeração do solo, fatores esses que inibem o desenvolvimento da planta (ALBUQUERQUE \& DURÃES, 2007).

Uma forma de controlar o desperdício da água na agricultura é estimar a necessidade hídrica da cultura, repondo a quantidade certa de água no solo e, assim, garantindo à planta o suprimento necessário para um bom desenvolvimento. $\mathrm{O}$ que tem dificultado o manejo da irrigação, principalmente em pequenas propriedades (agricultura familiar), é a falta de monitoramento dos dados meteorológicos para estimar a demanda hídrica das culturas. Os produtores não dispõem de capital financeiro para investirem na aquisição de uma estação meteorológica. De acordo com Elias et al. (2014), as estações meteorológicas profissionais geralmente têm alto custo e sua complexidade de operação restringe o seu uso.

Um sistema simples para aquisição de dados e para o controle automático da irrigação, quando disponível a baixo custo de aquisição, de manutenção, apresentando uma facilidade de uso por usuários comuns, evidencia uma boa receptividade do mercado e auxilia no desenvolvimento econômico de pequenos agricultores rurais que dispõem de amplas dificuldades em financiar mão de obra no processo de irrigação.

Uma plataforma com estas características, ou seja, um sistema de baixo custo para o controle e monitoramento da aquisição de dados é o Arduino. A plataforma Arduino tem sido empregada em pesquisas com diferentes finalidades (ELIAS et al., 2014; GALANTE \& GARCIA, 2014; GARCIA et al., 2015; SOUZA et al., 2015; TORRES et al., 2015).

Diante do exposto, objetivou-se com o presente trabalho desenvolver um protótipo de baixo custo de aquisição (plataforma Arduino) para monitoramento e controle automático de um sistema de irrigação, com acionamento remoto via aplicativo WEB.

\section{MATERIAL E MÉTODOS}

No presente trabalho foi desenvolvido um protótipo de baixo custo, nas seguintes etapas: construção do dispositivo físico (hardware), software (desenvolvido para abrir em qualquer navegador e sistema operacional Android) e testes em campo.

$\mathrm{Na}$ construção do dispositivo físico para automação do sistema de irrigação, foi utilizada uma placa de Arduino. O Arduino é um microcontrolador baseado no ATmega328. Dispõe de 14 pinos digitais de entrada/saída (dos quais 6 podem ser usados como saídas PWM - Pulse Width Modulation), 6 entradas analógicas, um ressonador cerâmico de $16 \mathrm{MHz}$, uma conexão USB, um jack para fonte, um cabeçalho ICSP, e um botão 
de reset. Contém o necessário para dar suporte ao microcontrolador, o usuário apenas o conecta a um computador com um cabo USB ou o liga com um adaptador AC-DC ou bateria (ARDUINO, 2015a).

Além do microcontrolador, utilizaram-se os sensores de temperatura e umidade relativa do ar DHT11 (disposto à sombra, protegido da chuva e fora da caixa de proteção do circuito para não apresentar valores imprecisos e para não danificálo) e sensor de chuva YL-83 (disposto à chuva e higienizado periodicamente - ao menos uma vez ao mês ou após períodos de chuva - para não expor valores inexatos), módulo relógio de tempo real RTC-DS1307 e como atuador uma válvula solenoide.

Com o sensor DHT11, obtiveram-se os valores de temperatura média, máxima e mínima do ar. O sensor YL-83 foi utilizado para quantificar o volume de água proveniente da chuva. Utilizouse o sensor RTC-DS1307 para sincronizar a data e horário do sistema com a data e horário do servidor Web ou com a data e horário sugerido pelo usuário. Utilizou-se um sensor de fluxo de água de meia polegada $(0,0127 \mathrm{~m})$, no qual suporta uma pressão máxima de 1,75 $\mathrm{MPa}$. Os valores são utilizados no microcontrolador por meio de sinal digital, para executar a função de fechamento da válvula solenoide. $\mathrm{O}$ atuador utilizado foi uma válvula solenoide de meia polegada $(0,0127 \mathrm{~m})$ que suporta uma pressão máxima de $0,8 \mathrm{MPa}$ e opera com tensão de até $12 \mathrm{~V}$. A função do atuador é de ejeção ou não da água utilizada para a irrigação.

Após a montagem do hardware realizou-se a programação da plataforma Arduino. Foi feito dentro do ambiente de programação próprio do Arduino, baseado no ambiente open-source, por meio de uma linguagem referente a este microcontrolador. $\mathrm{O}$ open-source faz com que seja fácil de escrever código e enviá-lo para a placa, executa no Windows, Mac OSX e Linux. O ambiente é escrito em Java e baseada em processing (linguagem de programação de código aberto e ambiente de desenvolvimento integrado - IDE) e outros softwares de código aberto. Este software pode ser usado com qualquer placa Arduino (ARDUINO, 2015b).

Todos os sensores utilizados operam com tensão de $5 \mathrm{~V}$ e os valores aferidos pelo microcontrolador são disponibilizados ao usuário do sistema através do servidor Web. Com exceção do sensor de chuva, todos os sensores operam com envio ou recebimento (no caso do sensor RTC-DS1307) de sinais analógicos.

Para a comunicação Web foi utilizado o circuito integrado HLK-RM04, no qual possui uma interface de comunicação serial para troca de dados com o microcontrolador ATmega328. Outra característica desse dispositivo é a simples configuração de conexão com uma rede WiFi através de uma página Web acessado a partir um endereço IP padrão. Todo o procedimento de configuração da conexão do dispositivo a uma rede WiFi é devidamente detalhado na página de monitoramento e configuração do sistema de controle automático de irrigação disponível ao usuário. $\mathrm{O}$ circuito integrado HLK-RM04 é responsável pela comunicação entre o microcontrolador e o servidor Web configurado, por meio de requisições HTTP e usando os protocolos TCP/IP.

O Transmission Control Protocol (TCP) e o Internet Protocol (IP) são dois dos protocolos mais importantes na Internet. $\mathrm{O}$ protocolo IP especifica o formato dos pacotes que são enviados e recebidos entre roteadores e sistemas finais. Os principais protocolos da Internet são conhecidos coletivamente como TCP/IP (KUROSE \& ROSS, 2012).

O HTTP é um protocolo usado principalmente para acessar dados na Web. Funciona como uma combinação de dois outros protocolos, FTP e SMTP. O HTTP é similar ao FTP, pois permite a transferência de arquivos e usa serviços do TCP. Entretanto, é muito mais simples que o FTP, pois usa uma única conexão TCP. Não existe uma conexão de controle separada, somente dados são transferidos entre o cliente e o servidor (FOROUZAN, 2006).

O projeto de controle automático do sistema de irrigação foi planejado e desenvolvido segundo os conceitos do paradigma de orientação a objetos (Object Oriented, OO), aos quais possuem estruturas de classes com atributos e métodos.

Classe é um conceito orientado a objeto que encapsula dados e abstrações procedurais necessárias para descrever o conteúdo e

\section{REVENG}


comportamento de alguma entidade do mundo real. Abstrações de dados que descrevem a classe são envolvidas por uma "parede" de abstrações procedurais capazes de manipular de certa maneira os dados (TAYLOR, 1998). Em uma classe bem projetada, a única maneira de atingir os atributos (e operar sobre eles) é passar por um dos métodos que formam a "parede". Portanto, a classe encapsula dados (dentro da parede) e o processamento que manipula os dados (os métodos que formam a parede). Isso possibilita o encapsulamento de informações e reduz o impacto de efeitos colaterais associados à mudança (PRESSMAN, 2011).

$\mathrm{Na}$ Figura 1 encontra-se um esboço das principais classes do Diagrama de Classes do projeto. O símbolo "[...]" indica que existe(m) outra(s) classe(s) atrelada(s) à(s) classe(s). Como atributos da classe "Irrigacao", têm-se: valvulaSolenoiteAberta (indica se a válvula está aberta ou não), modoManual (indica se o sistema operará no modo manual ou não) e vazaoAgua (indica volume de água ejetado pelo sistema). Como métodos, têm-se: definirModoManual() (ação executada pelo sistema/usuário que define a operabilidade do sistema - modo manual ou não), entre outros.

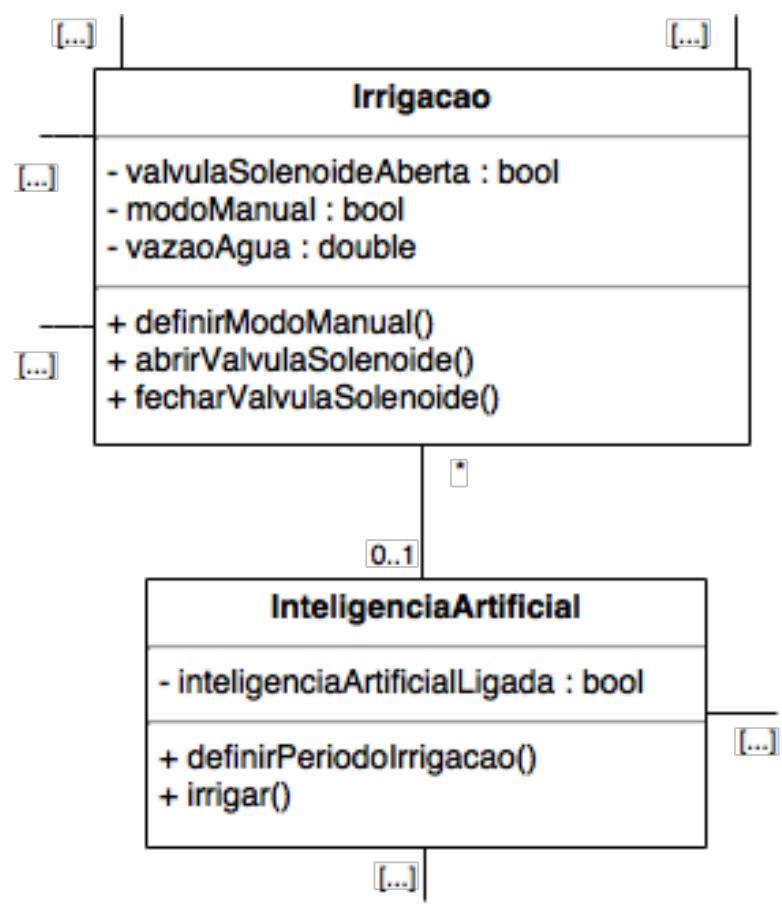

Figura 1. Parte do diagrama de classes do projeto para o controle automático de sistema de irrigação.
Os algoritmos adotados para o desenvolvimento do modo automático do sistema de irrigação foram constituídos a partir da evapotranspiração de referência estimada pelo método de HargreavesSamani (1985) (Equação 1). Tal método foi selecionado devido aos recursos disponíveis para implementação e custeio do sistema físico (hardware) (apenas sensor de temperatura), uma vez que se prezou na elaboração do projeto um hardware capaz de efetuar a maioria das aferições e atuações necessárias para o processo de irrigação e que fosse relativamente barato.

$$
\mathrm{ETo}=0,0023 \mathrm{R}_{\mathrm{a}}(T+1,8) \sqrt{\left(T_{\max }-T_{\min }\right)}
$$

em que,

ETo é a evapotranspiração de referência, em mm dia $^{-1}$

$\mathrm{R}_{\mathrm{a}}$ é a radiação no topo da atmosfera, em $\mathrm{MJ} \mathrm{m}^{-2}$ dia $^{-1}$ (obtida pelas Equações de 2 a 6);

$\mathrm{T}$ é a temperatura média diária do ar, em ${ }^{\circ} \mathrm{C}$;

$\mathrm{T}_{\max }$ é a temperatura da máxima diária do ar, em ${ }^{\circ} \mathrm{C}$; e

$\mathrm{T}_{\text {min }}$ é a temperatura mínima diária do ar, em ${ }^{\circ} \mathrm{C}$.

$$
\begin{aligned}
& \mathrm{R}_{\mathrm{a}}=37,586 d_{r}\left(\omega_{s} \sin (\varphi) \sin \left(\delta_{s}\right)+\cos (\varphi) \cos \left(\delta_{s}\right) \sin \left(\omega_{s}\right)\right) \\
& \mathrm{d}_{\mathrm{r}}=1+0,033 \cos \left(\frac{2 \pi}{365} J\right) \\
& \mathrm{J}=\operatorname{inteiro}(30,42 \cdot \mathrm{M}-15,23) \\
& \omega_{s}=\arccos \left(-\tan (\varphi) \tan \left(\delta_{s}\right)\right. \\
& \delta_{s}=0,4093 \sin \left(\frac{2 \pi}{365} J-1,405\right)
\end{aligned}
$$

em que,

$\mathrm{d}_{\mathrm{r}}$ é a distância relativa Terra-Sol, em radianos; $\omega_{\mathrm{s}}$ é o ângulo horário do pôr-do-sol, em radianos; $\varphi$ é a latitude local, em radianos;

$\delta_{\mathrm{s}}$ é a declinação solar, em radianos;

J é o dia Juliano do ano; e

M é o número do mês (1 a 12). 
A partir da estimativa da evapotranspiração de referência pelo método de Hargreaves-Samani (1985) associado ao coeficiente de cultura (Kc, adimensional), calculou-se a evapotranspiração da cultura (lâmina de irrigação a ser aplicada) (Equação 7). O Kc é tabelado e distinto para cada cultura e para cada estádio de desenvolvimento (MELLO \& SILVA, 2007).

$$
\mathrm{ETc}=\mathrm{EToKc}
$$

Como observado em outros trabalhos, o método de Hargreaves-Samani tem apresentado bons resultados em regiões onde há escassez de dados, sendo uma boa alternativa para evitar um grande desperdício de água no momento de aplicar uma determinada lâmina de irrigação (SILVA et al., 2015) e, por exemplo, atendeu satisfatoriamente à estimativa da ETo na região norte fluminense, RJ (MENDONÇA, 2003), contudo o método pode superestimar valores de evapotranspiração de referência (ETo), como se constatou no município de Rio Preto-MG (latitude de $22^{\circ}-05^{\prime}$-S, longitude de $43^{\circ}-49^{\prime} \mathrm{W}$ e altitude média de $430 \mathrm{~m}$ ) (SOUZA et al., 2001). Sendo os valores de evapotranspiração superestimados ou subestimados, dependendo da região da instalação do sistema de irrigação, os mesmos podem ser ajustados por regressão linear e/ou pela área do cultivo, uma vez que essas variáveis são multiplicadas entre si, como é demonstrado pela equação a seguir:

$$
\mathrm{V}_{\mathrm{ar}}=A p \cdot E T p c .1000-V_{a c a}
$$

em que,

$\mathrm{V}_{\boldsymbol{x}}$ é o volume de água requerida (em L), valor referência para ejeção de água pelo sistema de irrigação;

$A p$ é a área do plantio, $\mathrm{em} \mathrm{m}^{2} ; \mathrm{e}$

$\mathrm{V}_{\text {vaca }}$ é o volume de água da chuva, em $\mathrm{L}$ acumulados por dia.

O sistema Web de monitoramento e controle da irrigação foi desenvolvido na linguagem de programação PHP utilizando o banco de dados relacional MySQL disponível no servidor selecionado.

O MySQL pode ser considerado o mais popular SGBD (Sistema Gerenciador de Banco de Dados) SQL open source. Consiste em um banco de dados relacional. Funciona em Plataforma Windows, Linux, FreeBSD, BSDI, Solaris, Mac OSX, SunOS, SGI, etc. É compatível com drivers ODBC, JDBC, .NET, entre outros. O MySQL consiste apenas no SGBD e seu site oficial é o www.mysql.com (Miyagusku, 2008).

O sistema Web possui um bom nível de usabilidade com uma interface amigável, de fácil uso e de monitoramento dos dados disponibilizados pelo sistema físico de irrigação. O mesmo pode ser acessado de qualquernavegador Web ou através do aplicativo desenvolvido para o sistema operacional Android, assim o usuário também terá um cômodo acesso ao sistema de irrigação através de um dispositivo móvel que dispunha desse sistema operacional e do aplicativo em questão.

Munido de um identificador de usuário e senha, o usuário possuirá as seções, descritas nos próximos parágrafos, disponíveis para monitoramento e atuação no sistema de irrigação.

Home: página principal do sistema é possível visualizar os dados de temperatura e umidade relativa do ar, intensidade da chuva naquele momento (fraca, moderada ou forte), data e horário do sistema, o volume de água ejetado durante a irrigação daquele dia, as programações configuradas (no modo manual), o volume de água requerido para a irrigação e o volume de água acumulado da chuva (no modo automático) e se o dispositivo físico está conectado à Internet. Nessa página também é possível atuar no processo de irrigação, o usuário pode abrir ou fechar a válvula solenoide a qualquer momento, exceto durante a execução das tarefas programadas ou quando o modo automático estiver ativo. No modo manual, o usuário pode ditar em quais momentos o sistema deve operar através das programações rotineira e

\section{REVENG}


específica e pode ajustar a data e horário do sistema utilizando o servidor ou o seu ajuste manual. $\mathrm{O}$ usuário também pode ativar ou desativar o modo automático e quando ativo, o mesmo preenche um formulário com informações relevantes para o processo de irrigação no modo automático como: horário de preferência para execução do processo de irrigação, a área do plantio, o coeficiente da cultura $(\mathrm{Kc})$ tabelado na seção "Sobre a Irrigação" e a latitude da posição geográfica em que se encontra o cultivo (a latitude aproximada é sugerida pelo sistema com o valor calculado da posição em que o usuário acessou o sistema. Demais informações relevantes para o processo de irrigação no modo automático são aferidas e calculadas pelo microcontrolador.

Sobre a Irrigação: página que traz algumas informações relevantes sobre a técnica de irrigação, bem como uma tabela com valores do coeficiente de cultura $(\mathrm{Kc})$ para alguns tipos de cultivos em diferentes fases.

Sobre o Sistema: página que apresenta informações relevantes para o uso do sistema Web pelo usuário. A página traz também um formulário para que o usuário possa entrar em contato com o suporte técnico do sistema.

Gráficos: página que mostra alguns gráficos de dados coletados, como o consumo de água e temperatura média em determinados períodos. O usuário também pode filtrar os períodos que desejar.

Conexão WiFi: página que informa detalhadamente o processo conexão do sistema físico à rede WiFi para uso da Internet pelo mesmo.

Alterar Senha: página em que através de um breve formulário de senhas, o usuário pode alterar sua senha vigente.

Sair: desconecta o usuário do sistema.

O sistema de irrigação foi implantado em uma localidade pertencente ao município de Viana, ES ( $20^{\circ} 22^{\prime} \mathrm{S}, 40^{\circ} 28^{\prime} \mathrm{W}$ e $295 \mathrm{~m}$ de altitude). Na maior parte, o monitoramento automático do sistema de irrigação foi feito de Vitória, aproximadamente 23,7 km de distância da área de instalação do sistema.
A região selecionada para testes de campo apresentou algumas particularidades que facilitaram ou dificultaram o trabalho, são elas: a região apresentou condições climáticas, energéticas e hídricas favoráveis para coleta de dados e execução de alguns testes; para o cálculo da evapotranspiração da cultura de referência (ETo), utilizou-se de uma cultura que normalmente é utilizada como referência, a grama (MELLO \& SILVA, 2007); apesar dos problemas técnicos no decorrer da implantação do sistema como, por exemplo, o baixo sinal ou ausência do mesmo devido às cadeias montanhosas da região e à distância relativamente grande da antena de redes de comunicação de dados $3 \mathrm{G} / 4 \mathrm{G}$ de celular mais próxima, tais problemas foram sanados como no caso, com testes e utilização de antenas externas; a maior parte dos testes ocorreram remotamente devido à distância entre local de testes e a residência oficial do desenvolvedor.

As dimensões da área de instalação do sistema de irrigação foi de $17 \times 11 \mathrm{~m}$, totalizando 187 $\mathrm{m}^{2}$. Além da área irrigada, no modo automático (aplicativo para Android ou navegador de Internet) é requerido o coeficiente da cultura $(\mathrm{Kc})$, sendo a área em estudo cultivada com grama Esmeralda. A latitude do local é compreendia em aproximadamente $-0,3555235$ radianos.

Os testes foram conduzidos entre 28 de maço e 03 de julho de 2015, sendo o valor de Kc de 0,85 para o período compreendido entre 28 de maço a 01 de junho, Kc de 0,46 para o período compreendido entre 02 de junho a 03 de julho.

\section{RESULTADOS E DISCUSSÃO}

Ao acessar o aplicativo Web do sistema de controle automático de irrigação através do aplicativo desenvolvido para Android ou por um navegador de Internet, tem-se: a página de Login (Figura 2A), onde o usuário do sistema insere suas credenciais para ter acesso ao sistema; a página principal do sistema com a seção que apresenta os estados do sistema (Figura 2B), seção que 
A.
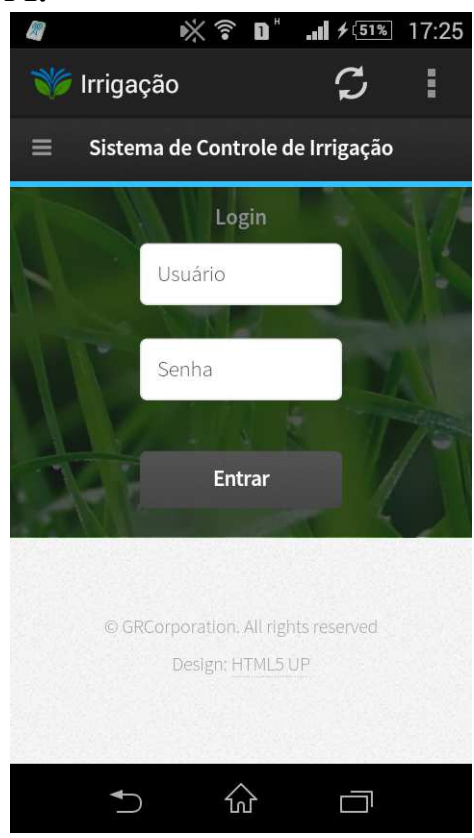

D.

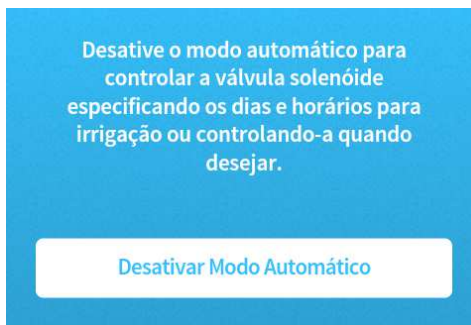

Informações disponibilizadas

1. pelo módulo físico de controle de irrigação Última data do sistema registrada: 03/08/2015 Último horário do sistema registrado: 17:25:25

Condições do ambiente e uso de água

Não está chovendo

Temperatura atual: $28^{\circ} \mathrm{C}$

Temperatura minima: $26^{\circ} \mathrm{C}$

Temperatura máxima: $29^{\circ} \mathrm{C}$

Temperatura média: $28^{\circ} \mathrm{C}$

Umidade relativa do ar: 55\%

Quantidade de água requerida: 361.55Litros

Acumulado estimado de água

de chuva: OLitros

Agua ejetada: 369.86 Litros
B.

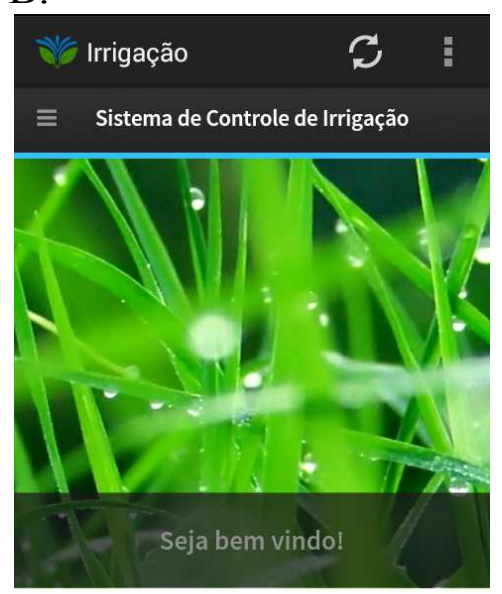

Módulo físico conectado à Internet?

A válvula solenóide está aberta?

Modo Automático ativo?

E.

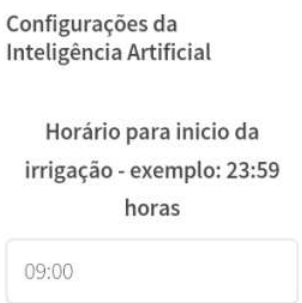

Tamanho da área do plantio exemplo: $1000 \mathrm{~m}^{2}$

100

Coeficiente de cultura (Kc) -

0.99

0.85

Latitude - exemplo: radianos

$-0.3541944$

Salvar
F.

C.
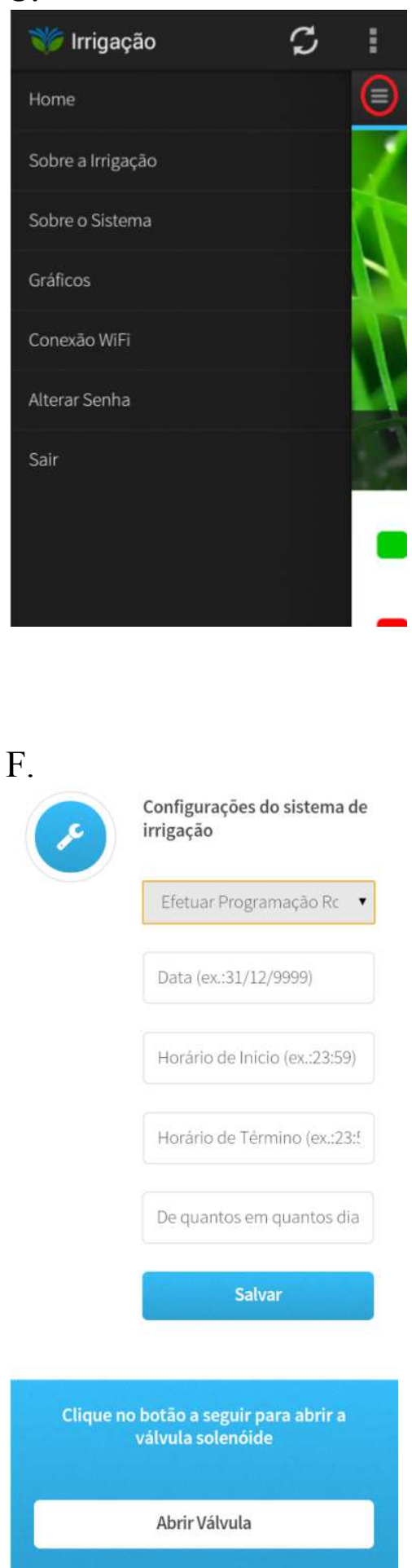

Figura 2. Página de Login (A); página principal do sistema com as seguintes seções: seção de estados (B), seção de links de acesso a demais páginas (acessada pressionando o símbolo circulado em vermelho) (C), seção com botão que serve para habilitar/desabilitar o sistema automático e seção com informações do sistema (D), seção com formulário para configuração do modo automático do sistema (E), seção com formulário para configuração do sistema em modo manual e seção para abertura/fechamento da válvula solenoide $(\mathrm{F})$. 
apresenta os links dos acessos de outras páginas do sistema (Figura 2C), seção das informações do sistema e seção do botão de ativar/desativar modo automático (Figura 2D), seção com formulário para configuração do modo automático do sistema (Figura 2E), seção para configuração do sistema em modo manual e seção de abertura/fechamento da válvula solenoide (Figura $2 \mathrm{~F}$ ).

Utilizando o aplicativo Network Signal Info (Kraemer, 2015) desenvolvido para Android, mediu-se a intensidade do sinal WiFi do local onde o módulo físico do sistema de irrigação foi instalado (Figura 3A). A distância entre o roteador e o módulo ficou em aproximadamente $10 \mathrm{~m}$ em linha reta e o sinal sofreu atenuação devido às paredes, móveis e eletrodomésticos dispostos entre os dispositivos. Constatou-se também que a intensidade do sinal de telefonia móvel da região sem o auxilio de uma antena externa (Figura 3B) não é suficiente para o estabelecimento de uma conexão com a Internet, desta forma foi necessário a instalação de uma antena externa de $17 \mathrm{~dB}(50,12 \mathrm{~mW})$ conectada à um modem de tecnologia $2 \mathrm{G} / 3 \mathrm{G} / 4 \mathrm{G}$. Uma vez que os dispositivos móveis (celulares) disponíveis não possuíam conectores para antena externa, não foi possível medir a intensidade do sinal da rede de telefonia móvel com o auxilio da antena externa instalada no local.

Para avaliar o funcionamento do sistema, foram obtidos dados de algumas irrigações ocorridas de forma programada com duração de $30 \mathrm{~min} \mathrm{dia}^{-1}$, duração aproximada para seguir a estimativa de $10 \mathrm{~L}$ de água por $\mathrm{m}^{2}$ para irrigação diária (Grama Brasil, 2015), bem como as condições do dia: temperaturas e chuva. Dias chuvosos com durações prolongadas (onde não se realizou irrigações), dias de manutenção do sistema e dias que ocorreram problemas de conexão (baixo sinal, expiração de créditos de dados disponibilizados pela operadora de telefonia móvel) foram desconsiderados. No Quadro 1, encontra-se o período monitorado com os respectivos valores do volume de água ejetado (modo manual - condição programada) e o volume de água necessário para irrigação calculada pelo sistema (modo automático).

Como a região é assistida por uma rede hidráulica comunitária com água proveniente de poços artesianos, o sistema foi instalado diretamente à rede, logo, devido às variações de pressão da água na rede, o volume de água ejetado entre os dias não apresentou muitas semelhanças quantitativas quando o sistema foi programado para efetuar a irrigação em um período de tempo durante um dia, $\left(30 \mathrm{~min} \mathrm{dia}^{-1}\right)$.

A partir das datas expostas no Quadro 1, a Figura 4 apresenta informações coletadas e calculadas pelo sistema: temperatura mínima, média e máxima (Figura 4A), umidade relativa do ar (Figura 4B), volume da água demandado e volume de chuva (Figura 4C) e volume de água ejetado em modo automático e programado (Figura 4D).

Apesar das diferenças entre os valores do volume de água ejetado pela condição programada do sistema, observa-se uma economia total de água de $26,80 \%$ no modo automático, podendo chegar a $61,27 \%$ com Kc igual a 0,46 , menor valor encontrado pelos experimentos de Silva (2004). Utilizando a estimativa de $10 \mathrm{~L}$ de água por $\mathrm{m}^{2}$ para irrigação em cada dia (Grama Brasil, 2015), tem-se uma economia total de água de 43,60\% com Kc igual a 0,85 e $70,16 \%$ com $\mathrm{Kc}$ igual a 0,46 .

Como o foco principal do trabalho foi o desenvolvimento de um sistema de baixo custo, $\mathrm{R} \$$ 197,08 para o protótipo cotado em julho de 2015, e de fácil utilização pelo usuário final, a análise de dados ficou limitada ao consumo de água a partir de poucas amostras de dados, sendo necessário um estudo aprofundado com maior tempo de coleta de dados para concluir algo da real economia hídrica utilizando o modo automático do sistema em relação aos modelos tradicionais de irrigação sem qualquer tipo de automação da irrigação. Outras avaliações que deverão ser feitas são a respeito do consumo de adubos e da redução do uso de mão de obra.

Uma demonstração da operação do sistema em modo manual está disponível no site: https://youtu. be/OHDmat7wCuE. 
A.

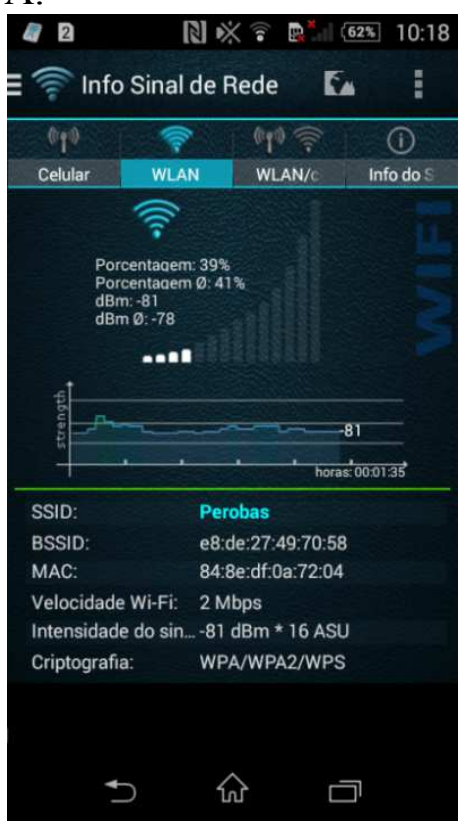

B.

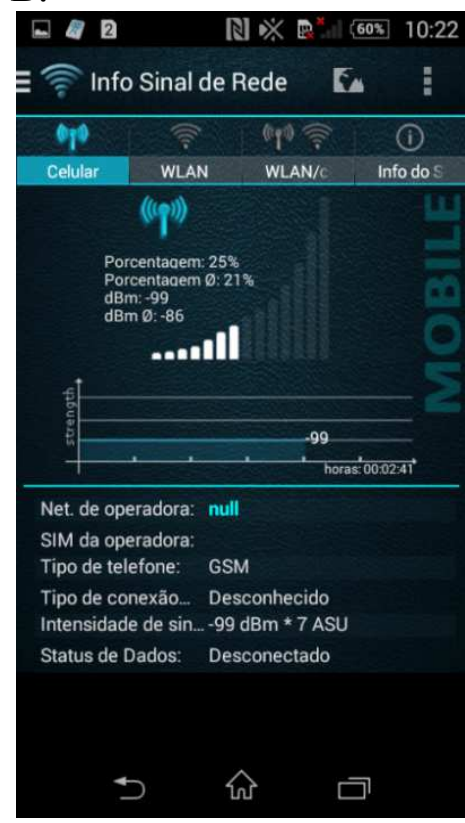

Figura 3. Intensidade do sinal WiFi do local da instalação do módulo físico do sistema de irrigação (A) e intensidade do sinal da rede de telefonia móvel sem o auxilio de antena externa (B).

Quadro 1. Volume de água ejetado para uma programação de $30 \mathrm{~min} \operatorname{dia}^{-1} \mathrm{e}$ volume de água requerido pelo modo automático do sistema.

\begin{tabular}{ccc}
\hline Data & $\begin{array}{c}\text { Volume de água ejetada }(\mathbf{L}) \\
\text { Programação: } \mathbf{3 0} \text { min dia }^{-1}\end{array}$ & $\begin{array}{c}\text { Volume de água requerida }(\mathbf{L}) \\
\text { Modo automático }\end{array}$ \\
\hline $28 / 03 / 2015$ & 1934,46 & 1190,12 \\
$30 / 03 / 2015$ & 2152,00 & 595,06 \\
$31 / 03 / 2015$ & 3877,00 & 1360,30 \\
$01 / 04 / 2015$ & 1657,23 & 1291,15 \\
$02 / 04 / 2015$ & 1998,96 & 1140,52 \\
$03 / 05 / 2015$ & 1315,50 & 1081,91 \\
$19 / 05 / 2015$ & 1232,40 & 373,82 \\
$23 / 05 / 2015$ & 433,97 & 1193,56 \\
$25 / 05 / 2015$ & 1438,49 & 1354,18 \\
$27 / 05 / 2015$ & 1122,28 & 1138,36 \\
$28 / 05 / 2015$ & 1366,30 & 1312,00 \\
$31 / 05 / 2015$ & 794,04 & 1027,83 \\
$02 / 06 / 2015$ & 1014,03 & 702,68 \\
$07 / 06 / 2015$ & 902,73 & 1083,31 \\
$09 / 06 / 2015$ & 866,49 & 1194,17 \\
$19 / 06 / 2015$ & 1254,28 & 941,09 \\
$01 / 07 / 2015$ & 1329,86 & 1046,55 \\
$03 / 07 / 2015$ & 1241,28 & 954,96 \\
\hline
\end{tabular}

\section{REVENG


A

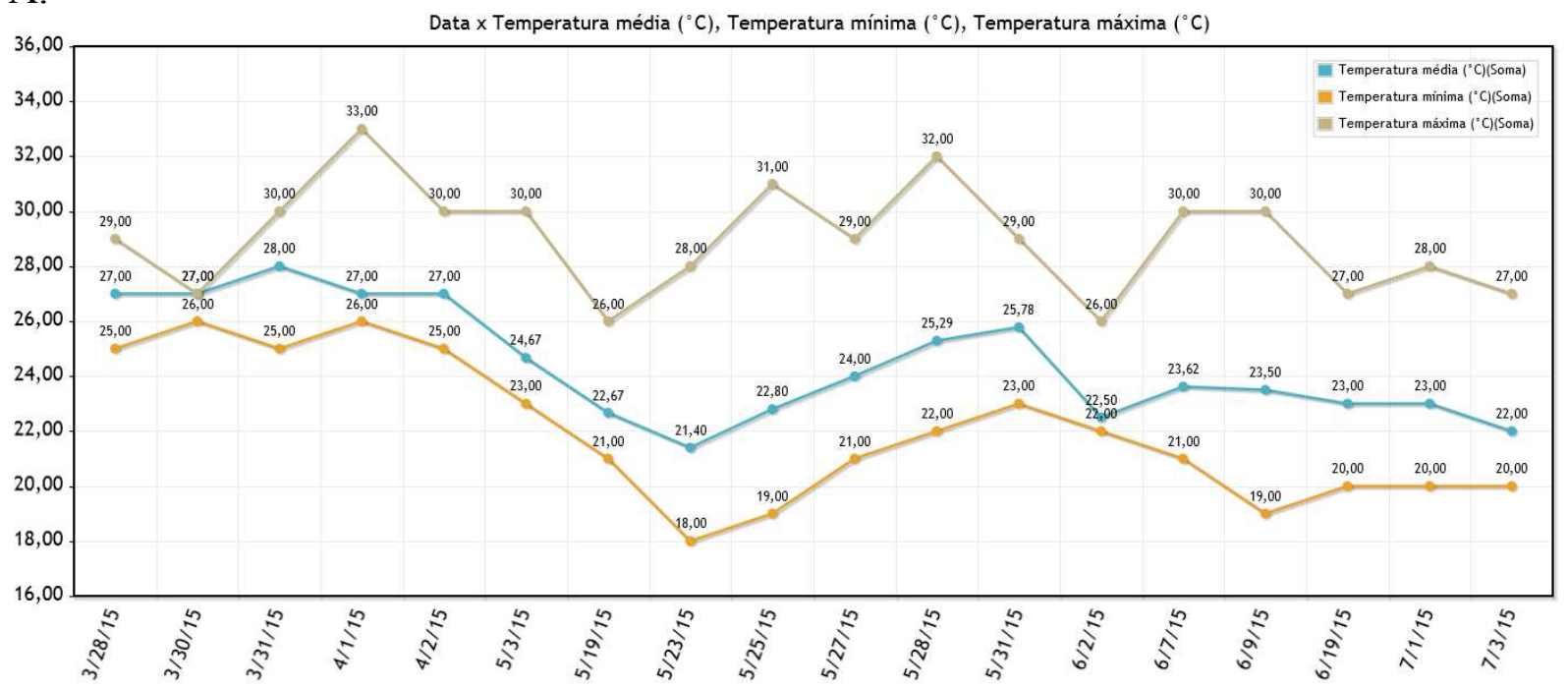

B.

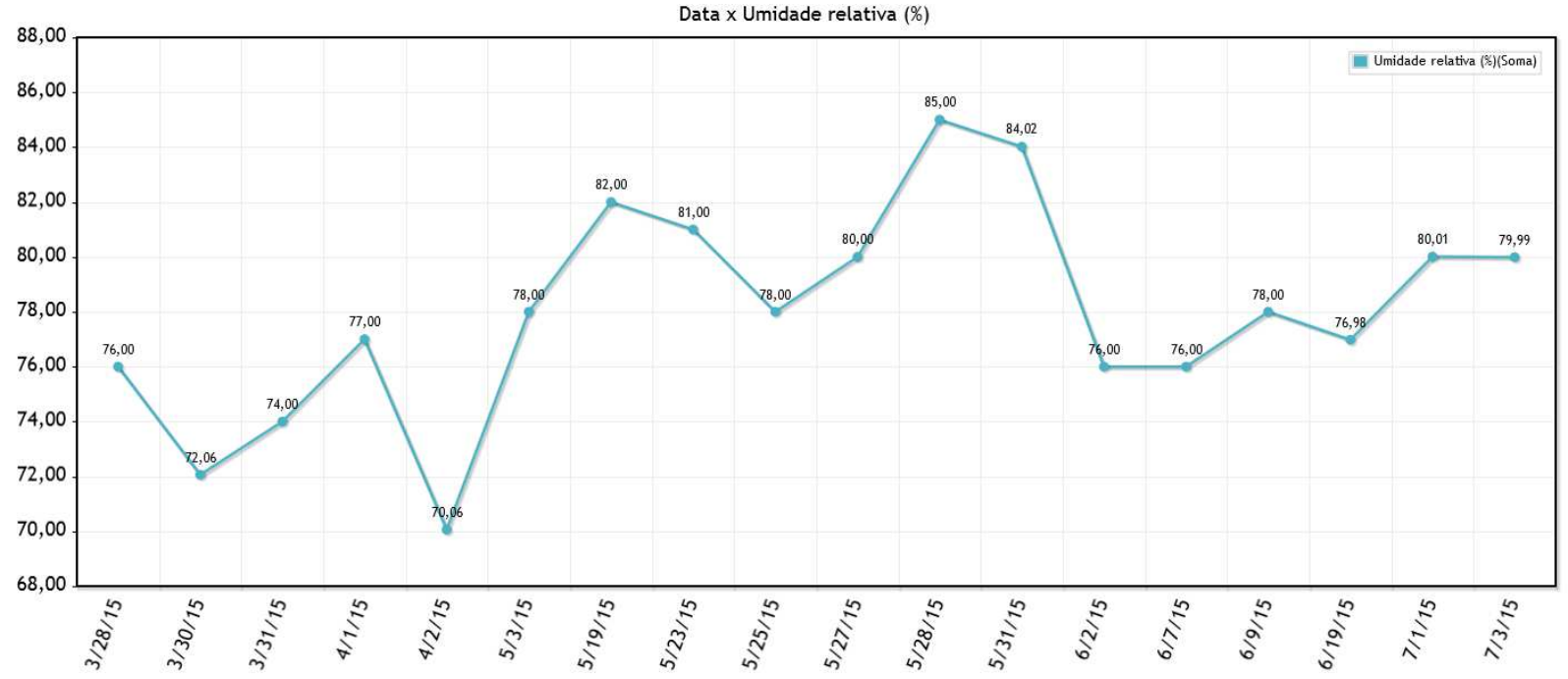

C.

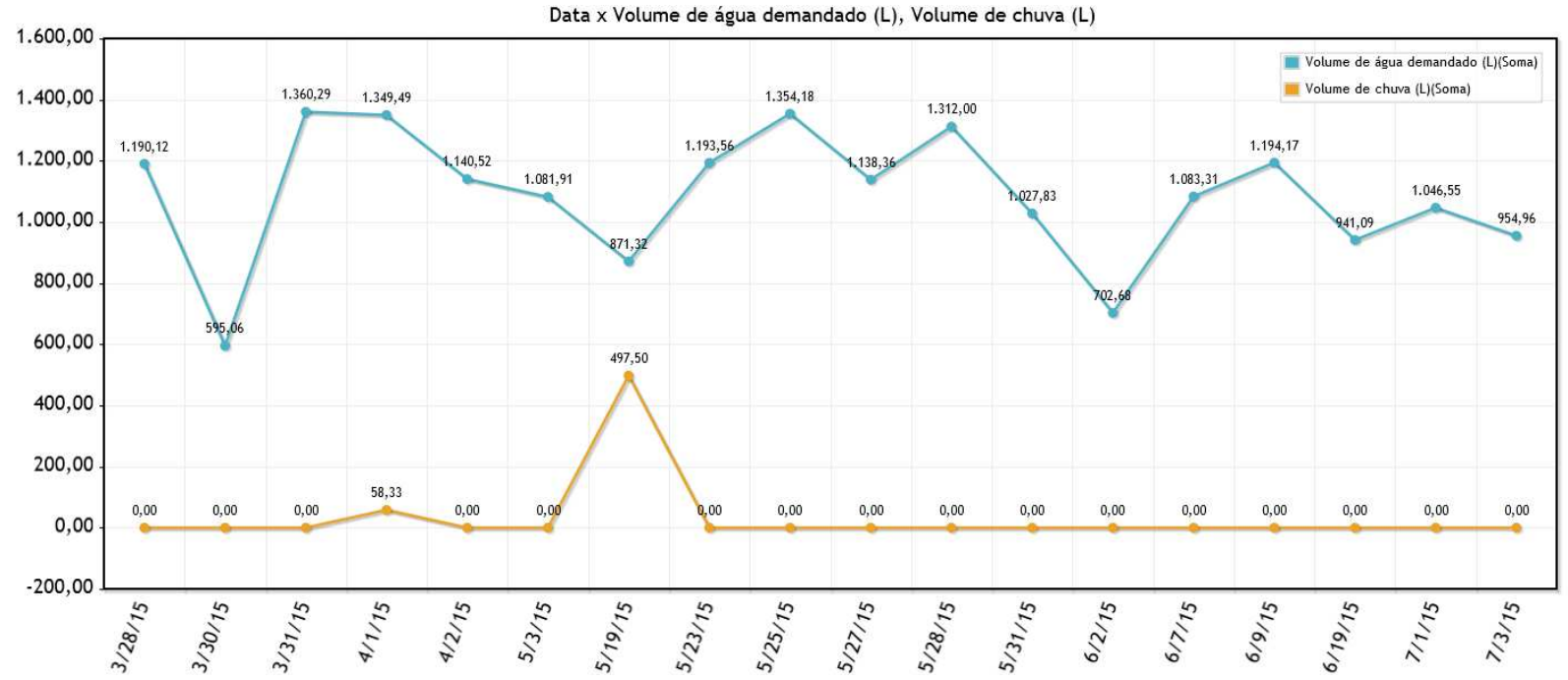




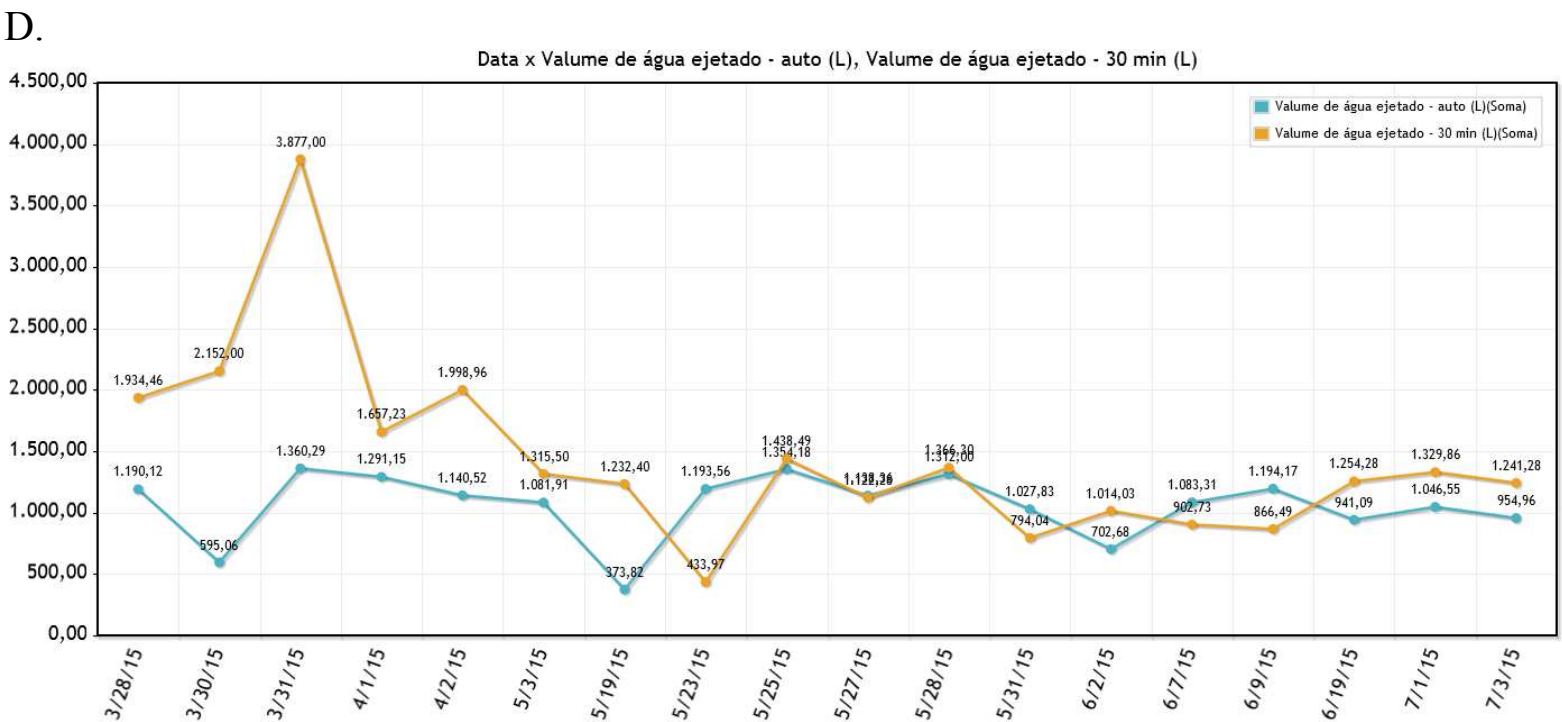

Figura 4. Gráfico de temperatura mínima, média e máxima (A), umidade relativa do ar (B), volume da água demandado e volume de chuva $(\mathrm{C})$ e volume de água ejetado em modo automático e programado (D).

\section{CONCLUSÕES}

Após testes de usabilidade com seis voluntários, evidenciou-se uma boa receptividade do sistema, sendo que estes não encontraram dificuldades em operar o sistema.

O baixo custo para o desenvolvimento do sistema viabilizou a promoção do projeto e consequentemente viabilizará um possível produto final.

Houve uma redução do consumo de água durante as irrigações em torno de $26,80 \%$ no modo automático do sistema.

\section{REFERÊNCIAS BIBLIOGRÁFICAS}

ALBUQUERQUE， P.E.P.; DURÃES， F.O.M. Uso e manejo de irrigação. Brasília: Embrapa Informação Tecnológica, 2007. 528p

ARDUINO. Arduino Uno.. . Disponível em: $\quad<$ https://www.arduino.cc/en/Main/ arduinoBoardUno $>$. Acesso em: 14 de julho de 2015a.

ARDUINO. Arduino Software.. . Disponível em: <https://www.arduino.cc/en/Main/Software>. Acesso em: 14 de julho de 2015b.
BERNARDO, S.; SOARES, A.; MANTOVANI, E. Manual de irrigação. 8.ed. Viçosa: Editora UFV, 2006.

ELIAS, A.A.A.; SILVA, J.C.P.; GONÇALVES, R.N.; SILVA, T.S. ArdWeather: Uma estação meteorológica baseada no Arduino e em Web Services RESTful. In: Safety Health and Environment World Congress, 14, 2014, CubatãoSP. Anais... Cubatão-SP, 2014. p.44-48.

FOROUZAN, B.A. Comunicação de dados e redes de computadores. 4.ed. Porto Alegre: AMGH Editora Ltda, 2008. 1168p.

GALANTE, A.C.; GARCIA, R.F. Sistema de aquisição de dados de sensores de baixo custo baseado no Arduíno. In: Congresso Brasileiro de Agricultura de Precisão, 6, 2014, São Pedro-SP. Anais... Jaboticabal: Associação Brasileira de Engenharia Agrícola, 2014.

GARCIA, R.F.; LIMA, R.C.; COIMBRA, C.M.M. Avaliação de um sensor de fluxo utilizando placa microcontroladora Arduino. Engenharia na Agricultura, Viçosa, v.23, n.1, p.162-168, 2015.

Grama Brasil. 2015. Disponível em: <http://www. 
brasilgramas.com.br/dicas>. Acesso em: 28 de julho de 2015.

\section{KRAEMER, A. Network Signal Info: KAIBITS}

Software. Versão xxxx Frankfurt, 2015. Disponível em: <https://play.google.com/store/ apps/details?id=de.android.telnet\&hl=pt_BR $>$. Acesso em: 08 de agosto de 2015.

KUROSE, J.F.; ROSS, K.W. Computer networking: a top-down approach. 6.ed. Upper Saddle River: Pearson, 2012. 864p.

MANTOVANI, E.C.; BERNARDO, S.; PALARETTI, L.F. Irrigação: princípios e métodos. 3. ed. Viçosa: Editora UFV, 2009. 355p.

MELLO, J.L.P.; SILVA, L.D.B. Irrigação. Universidade Federal Rural do Rio de Janeiro, Instituto de Tecnologia/Departamento de Engenharia, Rio de Janeiro, 2007. 180p.

MENDONÇA, J.C.; SOUSA, E.F.; BERNARDO, S.; DIAS, G.P.; GRIPPA, S. Comparação entre métodos de estimativa da evapotranspiração de referência (ETo) na região Norte Fluminense, RJ. Revista Brasileira de Engenharia Agrícola e Ambiental, Campina Grande, v.7, n.2, p.275-279, 2003.

MIYAGUSKU, R. Curso prático de SQL. São Paulo: Digerati Books, 2008. 96p.

PRESSMAN, R.S. Engenharia de Software: Uma Abordagem Profissional. 7.ed. Porto Alegre:
AMGH Editora Ltda, 2011. 780p.

SILVA, D.F. Análises quantitativa e qualitativa do crescimento e desenvolvimento da gramabatatais e grama-esmeralda em diferentes lâminas de irrigação. 2004. 62p. Dissertação (Mestrado em Engenharia Agrícola) - Universidade Federal de Viçosa, Viçosa, 2004.

SILVA, M.G.; OLIVEIRA, I.S.; CARMO, F.F.; LÊDO, E.R.F.; SILVA FILHO, J.A. Estimativa da evapotranspiração de referência pela equação de Hargreaves-Samani no Estado do Ceará, Brasil. Revista Brasileira de Engenharia de Biossistemas, Tupã, v.9, n.2, p.132-141, 2015.

SOUSA, F.H.F.; OLIVEIRA, B.R.A.; VIANA, T.P.; ESTÁCIO, P.M.; FREIRE, F.J.L. Sistema de produção agrometeorológico portátil utilizando a plataforma Uno. In: Congresso Técnico Científico da Engenharia e da Agronomia, 72, 2015, Fortaleza. Anais... Fortaleza, 2015.

SOUZA, L.O.C.; FACCIOLI, G.G.; MUDRIK, A.S.; MANTOVAN, E.C.I. Comparação da evapotranspiração de referência (ETo), estimada pelos modelos teóricos de Penman-Monteith e Hagreaves-Samani, com uso do Software SISDA. In: Simpósio de Pesquisa dos Cafés do Brasil, 2, 2001, Vitória. Anais... Embrapa Café: Brasília, 2001. p.506-511.

TAYLOR, D. Object-Oriented Technology: A Manager's Guide. 2. ed. Boston: Addison-Wesley Longman, 1998. 224p. 